\title{
A randomized controlled trial testing a hyaluronic acid spacer injection for skin toxicity reduction of brachytherapy accelerated partial breast irradiation (APBI): a study protocol
}

Gerson M. Struik ${ }^{1,2^{*}}$ (D) Jeremy Godart ${ }^{2}$, Gerda M. Verduijn ${ }^{2}$, Inger-Karine Kolkman-Deurloo ${ }^{2}$, Kim C. de Vries ${ }^{2}$, Raymond de Boer ${ }^{2}$, Linetta B. Koppert ${ }^{3}$, Erwin Birnie ${ }^{4,5}$, Ali Ghandi ${ }^{6}$, Taco M. Klem ${ }^{1}$ and Jean-Philippe Pignol ${ }^{2,7}$

\begin{abstract}
Background: Accelerated partial breast irradiation (APBI) is a treatment option for selected early stage breast cancer patients. Some APBI techniques lead to skin toxicity with the skin dose as main risk factor. We hypothesize that a spacer injected between the skin and target volume reduces the skin dose and subsequent toxicity in permanent breast seed implant (PBSI) patients.

Methods: In this parallel-group, single-center, randomized controlled trial, the effect of a subcutaneous spacer injection on skin toxicity among patients treated with PBSI is tested. Eligibility for participation is derived from international guidelines for suitable patients for partial breast radiotherapy, e.g. women aged $\geq 50$ years with a histologically proven non-lobular breast carcinoma and/or ductal carcinoma in situ (DCIS), tumor size $\leq 3 \mathrm{~cm}$, node-negative, and PBSI technically feasible. Among exclusion criteria are neoadjuvant chemotherapy, lymphovascular invasion, and allergy for hyaluronic acid. For the patients allocated to receive spacer, after the PBSI procedure, 4-10 cc of biodegradable hyaluronic acid (Barrige ${ }^{T M}$, Palette Life Sciences, Santa Barbara, CA, USA or Restylane SubQ ${ }^{\circledR}$, Galderma Benelux, Breda, the Netherlands) is injected directly under the skin using ultrasound guidance to create an extra $0.5-1 \mathrm{~cm}$ space between the treatment volume and the skin. The primary outcome is the rate of telangiectasia at two years, blindly assessed using Bentzen's 4-point scale. Secondary outcomes include: local recurrence; disease-free and overall survival rates; adverse events (pain, redness, skin/subcutaneous induration, radiation dermatitis, pigmentation, surgical site infection); skin dose; cosmetic and functional results; and health-related quality of life. A Fisher's exact test will be used to test differences between groups on the primary outcome. Previous studies found $22.4 \%$ telangiectasia at two years. We expect the use of a spacer could reduce the occurrence of telangiectasia to $7.7 \%$. A sample size of 230 patients will allow for a $10 \%$ lost to follow-up rate.

(Continued on next page)
\end{abstract}

* Correspondence: g.struik@franciscus.nl

1Department of Surgery, Franciscus Gasthuis and Vlietland, PO Box 10900,

3004 BA Rotterdam, The Netherlands

2Department of Radiation Oncology, Erasmus MC Cancer Institute, PO Box

5201, 3008 AE Rotterdam, The Netherlands

Full list of author information is available at the end of the article

(c) The Author(s). 2018 Open Access This article is distributed under the terms of the Creative Commons Attribution 4.0 International License (http://creativecommons.org/licenses/by/4.0/), which permits unrestricted use, distribution, and reproduction in any medium, provided you give appropriate credit to the original author(s) and the source, provide a link to the Creative Commons license, and indicate if changes were made. The Creative Commons Public Domain Dedication waiver (http://creativecommons.org/publicdomain/zero/1.0/) applies to the data made available in this article, unless otherwise stated. 
(Continued from previous page)

Discussion: In this study, the effect of a subcutaneous spacer injection on the skin dose, late skin toxicity, and cosmetic outcome is tested in patients treated with PBSI in the setting of breast-conserving therapy. Our results will be relevant for most forms of breast brachytherapy as well as robotic radiosurgery, as skin spacers could protect the skin with these other techniques.

Trial registration: Netherlands Trial Register, NTR6549. Registered on 27 June 2017.

Keywords: Breast neoplasms, Partial breast irradiation, Brachytherapy, Permanent breast seeds implant, Skin toxicity reduction, Telangiectasia, Spacer, Hyaluronic acid

\section{Background}

Breast cancer is increasingly diagnosed at an early stage $[1,2]$; for that stage, breast-conserving therapy, which includes wide local excision and radiotherapy, is equivalent to mastectomy in terms of local control and overall survival $[3,4]$. These oncological outcomes are excellent in early-stage breast cancer patients [2]. Hence, radiotherapy essentially provides a cosmetic and quality-of-life benefit over mastectomy [5]. Since local recurrences usually occur close to the primary tumor [6], the concept of accelerated partial breast irradiation (APBI) was introduced [7] to both reduce the amount of breast tissue irradiated and enable faster treatment. For well-selected patients, APBI has been tested and validated through large randomized clinical trials (RCT), using either brachytherapy [8-11], external 3D conformal radiotherapy [12, 13], or intraoperative radiotherapy $[14,15]$.

Brachytherapy has been the most evaluated technique and recent advances beyond multicatheter implantation include balloon or strut brachytherapy as well as permanent breast seed implants (PBSI) [16, 17]. Brachytherapy is generally well tolerated and reported long-term toxicities are acceptable. A lower incidence of low-grade acute skin toxicity for APBI, $21 \%$ vs $86 \%$ for whole breast radiotherapy $(p<0.001)$ has been reported for the GEC-ESTRO trial [18]. Regarding late side effects at five-year follow-up, lower rates of severe grade $2-3$ skin, $6.9 \%$ vs $10.7 \%$, and similar rates of subcutaneous side effects, $12.0 \%$ vs $9.7 \%$, were found in this study [19]. On the other hand, in a retrospective analysis of 1034 breast patients treated at The Ohio State University including 31\% treated with a balloon applicator, Wobb reported more seroma grade 2 or higher $(14.4 \%$ vs $2.9 \%, p<0.001)$, more painful fat necrosis $(10.2 \%$ vs $3.6 \%, p<0.001)$, and more telangiectasia grade 2 or higher $(12.3 \%$ vs $2.1 \%, p<0.001)$ for APBI compared to whole breast radiotherapy [20]. Among those permanent side effects, increased painful seroma is almost exclusively due to balloon applicator, fat-necrosis can be due to multiple factors, while telangiectasia is almost exclusively due to an excess of dose to the skin. This makes telangiectasia a specific marker of radiation toxicity [21, 22]. Telangiectasia corresponds to the dilation of an abnormal neo-vasculature in the skin following the destruction of normal capillaries by the radiation treatment, resulting in visible vessels [23]. Although rates are lower than with whole breast irradiation, in breast brachytherapy $10-27 \%[9,19,24]$ of the patients develop some grade of telangiectasia. The majority of lesions are grade 1 $\left(<1 \mathrm{~cm}^{2}\right)$ in breast radiotherapy studies reporting on late skin toxicity $[9,25,26]$. The onset of telangiectasia is from six months to 10 years after radiotherapy delivery [23]; however, rates of telangiectasia peak at two years with PBSI. Although permanent in most cases, some authors report disappearing of the telangiectasia with longer follow-up $[9,27]$. Nevertheless, if present, telangiectasia can remind patients of their cancer similar to a surgical scar and have a direct negative impact on the cosmetic outcomes $[9,26]$.

Several authors recommend keeping a distance of at least $5 \mathrm{~mm}$ between the planning target volume (PTV) and the skin $[28,29]$ and limiting the maximum skin dose to $70 \%$ [8]. However, such constraints are not always achievable. A simple solution would be the use of a spacer material injected subcutaneously to move the skin out of the high dose region [21].

In this manuscript, we report the protocol of a RCT investigating the clinical benefit of a subcutaneous spacer injection on the skin dose, late skin toxicity, and cosmetic outcome in patients treated with low dose rate (LDR) seed brachytherapy. For this study, the breast skin is considered as a critical structure for the radiotherapy and the clinical outcomes are measured using a breakdown of traditional skin toxicity scales in order to capture the toxicity that is specific to radiotherapy $[9,21,23,30]$.

\section{Methods/design}

\section{Aim and design}

We propose a parallel-group RCT comparing the occurrence of telangiectasia at two years in PBSI patients with or without a subcutaneous spacer injection. Allocation ratio is $1: 1$ and the trial is designed to test the superiority of the intervention. The primary hypothesis for the trial assumes that an injected hyaluronic acid spacer will reduce skin dose of PBSI and eventually the rate of telangiectasia at two years, compared to patients undergoing PBSI without spacer. As the intervention is applied when 
the patient is sedated, a placebo injection as comparator was deemed unnecessary. The methods section is described according to the Standard Protocol Items: Recommendations for Interventional Trials (SPIRIT) 2013 checklist (see Additional file 1).

Eligible patients will be recruited at hospitals referring patients after breast-conserving surgery for adjuvant radiotherapy at the Erasmus MC Cancer Institute, a large University hospital in Rotterdam, where the PBSI technique can be performed in the Netherlands.

\section{Eligibility and exclusion criteria}

Eligibility criteria were derived from international guidelines $[31,32]$ for suitable patients for partial breast radiotherapy. Eligible patients are females aged $\geq 50$ years with a confirmed histological diagnosis of invasive ductal carcinoma (IDC) and/or papillary, tubular, cribriform or medullar carcinoma and/or ductal carcinoma in situ (DCIS), after breast-conserving surgery with axillary node dissection (with a minimum of six nodes sampled) or sentinel lymph node biopsy. The maximum dominant tumor size is $3 \mathrm{~cm}$ and the tumor must be excised with negative surgical margins at ink for invasive carcinoma and $\geq 2 \mathrm{~mm}$ negative margins for DCIS or have a negative re-excision. The PBSI should be deemed technically feasible based on the seroma location, visibility, and size performing an ultrasound; the total implanted volume should be $<150 \mathrm{cc}$. Patient should have signed an informed consent.

Ineligible patients include those with lymphovascular invasion, lobular features on histology (pure or mixed) or sarcoma histology, triple negative tumors, extensive in situ carcinoma, multicentric disease (in more than one quadrant or separated by $\geq 2 \mathrm{~cm}$ ), bilateral breast cancer, recurrent breast cancer, Paget's disease of the nipple, metastases or active other cancer (defined by any malignancy in $<5$ years, excluding any cured non-melanoma skin cancer or cervical cancer), neoadjuvant chemotherapy, known allergy for hyaluronic acid, active auto immune disorder with severe vasculitis component, uncontrolled and complicated insulin-dependent diabetes, pregnancy, cosmetic breast implants, psychiatric or addictive disorder that would preclude attending follow-up, or postoperative wound infection or abscess following Centers for Disease Control and prevention (CDC) criteria.

\section{Interventions}

The permanent seed implant procedure includes a computed tomography (CT) simulation done positioning the patient similarly to for external beam breast radiotherapy. The clinical target volume (CTV) corresponds to the seroma with a 1-cm margin, limited to the fascia pectoralis, and $5 \mathrm{~mm}$ below the skin; the planned target volume (PTV) is an additional expansion of $0.5 \mathrm{~cm}$ with the same skin and chest wall limits. A pre-implant plan is generated using the MIM Symphony software (MIM Software Inc., Cleveland, OH, USA) to order stranded ${ }^{103} \mathrm{Pd}$ seeds of $2.5 \mathrm{U}$ activity.

For the procedure, anesthesia includes a non-steroidal anti-inflammatory drug (NSAID) for two days, light sedation with Propofol, and local freezing. Patients are positioned on a breast board, with the arm abducted similarly to the CT simulation. The breast skin is disinfected and the patient draped. The PTV projection perpendicular to the fiducial needle axis is outlined on the skin surface and verified using ultrasound. A PBSI template (Concure Oncology, Seattle, WA, USA) is attached to the fiducial needle and immobilized using a modified medical articulated arm (Fisso, Medtec Baitella Alt, Switzerland). The preloaded needles containing ${ }^{103} \mathrm{Pd}$ strands are then inserted under US guidance [33]. In patients allocated to receive spacer, an amount of 4-10 cc of biodegradable hyaluronic acid (Barrigel ${ }^{\mathrm{Ts}}$, Palette Life Sciences, Santa Barbara, CA, USA or Restylane SubQ ${ }^{\oplus}$, Galderma Benelux, Breda, the Netherlands) is injected directly under the skin under ultrasound guidance covering the PTV projection, aiming to create an extra $0.5-1 \mathrm{~cm}$ space between the treatment volume and the skin. If the skin is judged not to be at risk in all projection quadrants, it could be decided to only inject the area at risk. The injected skin quadrants will be reported specifically. All radiation oncologists involved in this study are trained to perform the intervention and the injection procedure and reporting instructions are incorporated in trial protocols. The hyaluronic acid spacer is expected to be fully degraded after 3-9 months.

\section{Outcomes}

The primary endpoint of this trial is the occurrence of telangiectasia at two years after PBSI. Assessment is performed by a blinded physician, following the Bentzen's 4-point scale which is included in the LENT/ SOMA questionnaire [34, 35]. This scale is defined as: "none;" grade I, "< $1 \mathrm{~cm}^{2}$," grade II, " $1-4 \mathrm{~cm}^{2}$;" and grade III, "> $\mathrm{cm}^{2}$." Patients will also be blinded for the allocated treatment. The secondary outcomes include the local recurrence rate at five and ten years, the disease-free and overall survival rates at five years, as well as brachytherapy and spacer injection adverse events (AE) according to the commonly used NCI Common Toxicity Criteria for Adverse Events (CTCAE v 4.03) scale for acute side effects [36], practically occurring within three months of the brachytherapy, and the Radiation Therapy Oncology Group (RTOG)/ European Organisation for Research and Treatment of Cancer (EORTC) scoring systems for late side effects [37], practically occurring after three months. The symptoms include the experience of pain, skin redness, pigmentation, induration, dermatitis, subcutaneous induration, and the occurrence of infection at the site of spacer injection. 
Additionally, patient-reported outcome measures (PROMs) include the cosmetic result with the Breast Cancer Treatment Outcome Scale (BCTOS) questionnaire [38], using a validated Dutch version which will shortly be published by our group [39] and the health-related Quality of Life using the Dutch version EORTC QLQ-C30 and BR23 questionnaires, version 3 [40]. Ipsilateral breast recurrence must be proven getting a copy of the biopsy or the salvage surgery pathology report. Dosimetry outcomes include the PTV volumes receiving at least $100 \%$ or $200 \%$ of the prescribed dose $\left(\mathrm{V}_{100}\right.$ and $\left.\mathrm{V}_{200}\right)$ as quality assurance for all treatment plans and maximum dose to a small skin volume of $2 \mathrm{~mm}$ thickness over $1 \mathrm{~cm}^{2}\left(D_{0.2 \mathrm{cc}}\right)[21,41]$ and the presence of a hotspot (isodose $\geq 90 \%$ ) [42] on $1 \mathrm{~cm}^{2}$ of the skin as indicators of skin toxicity risk.

Outcomes are collected before the PBSI implantation at baseline, at the end of the procedure, and at two months, six months, and every year up to five years, during follow-up visits at the cancer center. If a patient does not attend a follow-up appointment, she will be called and/or her family doctor contacted. Reason for no-show will be recorded in order to ensure exhaustive capture of survival, recurrence, and/or AEs. Overall and diseasespecific survival will be assessed until 10 years through GBA (Population registry, Gemeentelijke Basis Administratie) and/or general practitioners. A summary of the timing of questionnaires is detailed in Fig. 1.

\section{Sample size}

Previous studies found $22.4 \%$ telangiectasia at two years [9]. We expect the use of a spacer could reduce the skin dose to $50 \%$ [21] and the occurrence of any telangiectasia ( $\geq$ grade 1 ) to $7.7 \%$ [43]. To test this reduction (e.g. the superiority of the intervention), 105 (Fisher's exact test) patients per treatment arm would be needed $(\alpha=0.05$, $\beta=0.20$ ). A sample size of 230 patients will allow for a $10 \%$ lost to follow-up rate.

\section{Recruitment}

The Erasmus MC - Cancer Institute treats approximately 1000 patients with adjuvant breast radiotherapy every year. Given the inclusion criteria, it is expected that approximately $20 \%$ of these patients are eligible for PBSI. On top of this, referrals from outside the area are also expected specifically for PBSI. This makes it very likely that the required sample size could be recruited in three years.

\section{Treatment allocation and blinding}

After written informed consent and final eligibility check, the radiation oncologist will enroll the patient and randomization will be performed by the department's independent trial manager. Patients will be randomly allocated to one of the treatment arms (spacer injection or no spacer injection) in a 1:1 allocation ratio, applying a variable block size randomization (block sizes 2, 4, and 6). This concealed allocation will be computer-generated using the online randomization tool ALEA.

Patients will be blinded for the allocated treatment, as the spacer injection is performed under sedation. However, in some cases the patient might see or feel the effect of the spacer injection later. The treating radiation oncologist will be blinded during treatment planning and during the implant of the palladium seeds and be unblinded after the implant to inject the spacer or not using a telephone call with the departments trial manager.

Investigators will be blinded for allocated treatment during assessment of primary endpoint by performing this assessment in a separate visit in which the physician does not have access to the patient's medical file. Unblinding will be performed if a patient is going (un)planned off-study. In addition, in case of medical emergencies possibly caused by the spacer, unblinding will be performed. In these cases, a patient's allocated treatment can be unblinded by checking the medical record of the implantation or by contacting the trial management.

\section{Data management}

Secure collection of data is performed. Data entry will be performed using a predefined case report form (CRF) (Additional file 2) with accompanied data entry protocol. This provides instructions units to be used, missing data handling and range checks.

\section{Statistical methods}

All statistical tests will be two-sided and a $p$ value $<0.05$ is considered to be significant. Statistical analyses will be performed using IBM SPSS version 24 (IBM Corporation, Armonk, NY, USA). Data will be analyzed following intention-to-treat and per-protocol. Missing data will be handled using multiple imputation. Descriptive statistics will be used for all outcome measures.

A Fisher's exact test or Chi-squared test will be performed to test the reduction in the rate of telangiectasia in the study groups at two-year follow-up, i.e. to test the hypothesis that the rates of telangiectasia in both study groups are equal (superiority study).

Local recurrence-free survival as well as overall and disease-specific survival rates at five and ten years will be estimated using the Kaplan-Meier method. The local recurrence rate will be reported at five and ten years. A Fisher's exact test or Chi-squared test will be performed to test the difference in proportions (six-month, oneyear and two-year cumulative rate of side effects, skin dose $>90 \%$ over at least $1 \mathrm{~cm}^{2}$ at post-planning) between groups. (Skin) dosimetry data will be compared using a Mann-Whitney U test or an unpaired Student's t-test depending of distribution of data. To study the effect of 


\begin{tabular}{|c|c|c|c|c|c|c|c|c|}
\hline \multirow[b]{3}{*}{ TIMEPOINT } & \multicolumn{8}{|c|}{ STUDY PERIOD } \\
\hline & \multirow{2}{*}{$\begin{array}{c}\text { Enrolment } \\
\qquad-t_{1}\end{array}$} & \multirow{2}{*}{$\begin{array}{c}\text { Allocation } \\
0\end{array}$} & \multicolumn{5}{|c|}{ Post-allocation } & \multirow{2}{*}{$\begin{array}{c}\begin{array}{c}\text { Close- } \\
\text { out }\end{array} \\
10 \text { year }\end{array}$} \\
\hline & & & $\begin{array}{c}2 \\
\text { months }\end{array}$ & $\begin{array}{c}6 \\
\text { month } \\
s\end{array}$ & $\begin{array}{c}1 \\
\text { year }\end{array}$ & $\begin{array}{c}2 \\
\text { year }\end{array}$ & $\begin{array}{c}3-5 \\
\text { year }\end{array}$ & \\
\hline \multirow{5}{*}{$\begin{array}{r}\text { ENROLMENT: } \\
\text { Eligibility screen } \\
\text { Informed consent } \\
\text { Technical } \\
\text { feasibility } \\
\text { screening (US/CT) } \\
\text { Allocation }\end{array}$} & & & & & & & & \\
\hline & $\mathrm{X}$ & & & & & & & \\
\hline & $\mathrm{X}$ & & & & & & & \\
\hline & $X$ & & & & & & & \\
\hline & & $\mathrm{X}$ & & & & & & \\
\hline \multicolumn{9}{|l|}{ INTERVENTIONS: } \\
\hline $\begin{array}{r}\text { arm A: PBSI } \\
\text { without spacer }\end{array}$ & & $\mathrm{X}$ & & & & & & \\
\hline $\begin{array}{r}\text { arm B: PBSI with } \\
\text { spacer }\end{array}$ & & $X$ & & & & & & \\
\hline \multirow{3}{*}{$\begin{array}{r}\text { ASSESSMENTS: } \\
\text { Patient and tumor } \\
\text { characteristics } \\
\text { Baseline clinical } \\
\text { assessment }\end{array}$} & & & & & & & & \\
\hline & $X$ & $X$ & & & & & & \\
\hline & $X$ & & & & & & & \\
\hline \multirow{2}{*}{$\begin{array}{r}\text { Pre-implant QA } \\
\text { PBSI+spacer } \\
\text { procedure data }\end{array}$} & & $X$ & & & & & & \\
\hline & & $X$ & & & & & & \\
\hline \multirow{4}{*}{$\begin{array}{r}\text { Post-implant QA } \\
\text { Primary outcome } \\
\text { assesment } \\
\text { Adverse } \\
\text { events/side } \\
\text { effects } \\
\text { Survival and } \\
\text { recurrence } \\
\end{array}$} & & & $\mathrm{X}$ & & & & & \\
\hline & & & & & & $x$ & & \\
\hline & & & $\mathrm{X}$ & $\mathrm{X}$ & $\mathrm{X}$ & $X$ & $\mathrm{X}$ & \\
\hline & & & $X$ & $X$ & $\mathrm{X}$ & $\mathrm{X}$ & $\mathrm{X}$ & $\mathrm{X}$ \\
\hline \multicolumn{9}{|l|}{ Questionnaires } \\
\hline встOS & $x$ & & $x$ & & $x$ & $x$ & & \\
\hline EORTC QLQ-C3O & $x$ & & & $x$ & $x$ & $x$ & $x$ & \\
\hline EORTC QLQ-/BR23 & $x$ & & & $x$ & $x$ & & & \\
\hline$E Q-5 D$ & $x$ & & & & $x$ & $x$ & $x$ & \\
\hline
\end{tabular}

Fig. 1 Schedule of enrolment, interventions, and assessments in this study

spacer on cosmesis (BCTOS questionnaire) and quality of life (EORTC-QLQ-C30/BR23 and EQ5-D questionnaire) over time, a repeated measurements analysis will be performed (linear mixed model, covariance structure: unstructured) with independent variables time, allocated group, and interaction effects between time and allocated group.

\section{Discussion}

For early stage breast cancer patients that have outstanding survival outcomes $[3,4]$, the role of radiotherapy is essentially cosmetic [5]. The skin is a critical structure in breast radiotherapy, with skin dose as the main risk factor [21, 22]. In this study, we test an intervention to reduce cosmetic impairment by aiming to prevent long-term skin toxicity.
Telangiectasia are a specific marker of radiation toxicity [21, 22]. Although rates are lower than with whole breast irradiation, in breast brachytherapy $10-27 \%[9,19,24]$ of the patients develop some grade of telangiectasia. Rates of telangiectasia normally peak at two years till it stabilizes. Most of the lesions are permanent resulting in decreased quality of life [9]. Other skin toxicity scales (pigmentation, induration, fibrosis) are less specific for capturing radiation induced side effects [21].

Among our secondary outcomes are standard oncological outcomes. Based on our pre-clinical study we do not expect the spacer to influence the oncological effectivity of PBSI. [44]. This work in mastectomy specimens showed excellent feasibility of creating an extra 5-mm space directly below the skin using a biodegradable spacer. This space is not part of the PTV in LDR seed 
brachytherapy as the CTV expansion is limited to $5 \mathrm{~mm}$ below the skin by protocol [33]. The spacer partly lifted the skin, but also moved the breast tissue inferior and laterally. However, with the seeds already in place, we expect that any change in PTV geometry will be similar in the treated volume containing the seeds. This hypothesis was supported by the excellent and comparable PTV coverage $\left(\mathrm{V}_{100 \%}\right)$ before and after injection in the pre-clinical study [44]. However, this finding should be confirmed in a clinical setting.

Other secondary outcomes are brachytherapy and spacer injection AEs according to commonly used NCI CTCAE and RTOG/EORTC scoring systems for late side effects. Where our main hypothesis is that the spacer increases distance and reduces skin dose and telangiectasia, this will allow for analyzing the effect on other less radiotherapy-specific symptoms such as pain, skin redness, pigmentation, induration, dermatitis, subcutaneous induration, and the occurrence of infection at the site of spacer injection. Although hyaluronic acid is widely used as a dermal filler, the application as a skin spacer in patients treated with breast radiotherapy is a new concept and any unexpected side effects will be captured. Skin dose outcomes will potentially lead to updated skin dose constraints in treatment planning. Also, it could distinguish radiotherapy induced toxicity from other causes (i.e. intervention-related toxicity). PROMs assess the effect of the skin spacer on cosmesis, function, and quality of life. Furthermore, by using internationally recognized PROMs, a better comparison with other radiotherapy techniques is possible.

This clinical trial was designed because it is unknown whether the dosimetric benefit of the spacer, which was found in our pre-clinical study [44], translates in a real patient benefit. An example of a clinical trial that could not demonstrate that a dosimetric benefit translates into better patient outcomes, is the breast intensity modulated radiation therapy (IMRT) trial. In this trial, the improved radiation dose distribution and reduced moist desquamation using IMRT, compared to standard wedge $\mathrm{RT}$, did not result in reduced long-term side effects such as chronic breast pain [43].

Our primary analysis will be done following the intention-to-treat principle: the effect of skin spacer on telangiectasia rate at two years. However, a per-protocol analysis will allow for a better definition of a successful skin spacer as the skin spacer injection protocol $(>5 \mathrm{~mm}$ in a PTV skin projection area) is not definite and the trial could be hypothesis-generating.

A drawback of our study is that we are not able to secure a full double-blind design. Patients might be aware of an injected spacer as it could be palpable under the skin. For physicians, it might be possible to remember the allocated treatment after being unblinded during the
PBSI procedure. However, with the assessment of the primary outcome at two-year follow-up, this memory effect is not likely to cause any bias. Also, the type of outcome measure (telangiectasia using Bentzen's 4-point scale) allows for an objective, reproducible assessment. Furthermore, this a single-center study and our findings should be confirmed in a multicenter setting. Lastly, with only patients undergoing PBSI in this study, generalization of our findings to other APBI techniques should be done with caution. However, theoretically, this principle would hold for any APBI technique with a rapid dose fall off.

In this trial, we investigate the effect of a subcutaneous spacer injection on the skin dose, late skin toxicity, and cosmetic outcome in patients treated with PBSI in the setting of breast-conserving therapy. Our results will be relevant for most forms of breast brachytherapy as well as robotic radiosurgery, as skin spacers could be used to protect the skin with these other techniques.

\section{Trial status}

Protocol version 5, 26 March 2018. The first patient was enrolled in the study on 8 September 2017. Expected completion of recruitment is at the end of 2020 .

\section{Additional files}

Additional file 1: SPIRIT 2013 checklist. Recommended items to address in a clinical trial protocol and related documents. (PDF $117 \mathrm{~kb}$ )

Additional file 2: CRF template. A predefined case report form provides instructions units to be used, missing data handling and range checks. (PDF $415 \mathrm{~kb}$ )

\begin{abstract}
Abbreviations
APBI: Accelerated partial breast irradiation; BCTOS: Breast Cancer Treatment Outcome Scale; CDC: Centers for Disease Control and Prevention; CRF: Case report form; CT: Computed tomography; CTCAE: Common Toxicity Criteria for Adverse Events; CTV: Clinical target volume; $D_{0.2 c c}$ : Maximum dose to a small skin volume of 0.2cC; DCIS: Ductal carcinoma in situ; EORTC: European Organisation for Research and Treatment of Cancer; GEC-ESTRO: Groupe Européen de Curiethérapie and European SocieTy for Radiotherapy \& Oncology; HA: Hyaluronic acid; IDC: Invasive ductal carcinoma; IMRT: Intensity modulated radiation therapy; LDR: Low dose rate; LENT/SOMA: Late effects of normal tissues, subjective, objective, management, analytical; MREC: Medical research ethics committee; NSAID: Non-steroidal anti-inflammatory drugs; PBSI: Permanent breast seed implant; PROM: Patient-reported outcome measure; PTV: Planned target volume; RTOG: Radiation Therapy Oncology Group; SPIRIT: Standard Protocol Items: Recommendations for Interventional Trials; US: Ultrasound; $V_{100}$ : Volume of PTV receiving at least $100 \%$ of prescribed dose; $V_{200}$ : Volume of PTV receiving at least $200 \%$ of prescribed dose
\end{abstract}

\section{Acknowledgements}

Not applicable.

\section{Funding}

GS receives a financial research support through the Theia Foundation (no. 2016244), the Coolsingel Foundation (no. 444), and the Franciscus Friends Fund (Brachytherapy for Breast Cancer Research Project). The funders had no role in study design, data collection and analysis, decision to publish, or preparation of the manuscript. 


\section{Availability of data and materials}

In order to maximize health information protection, patient data will be captured using standard anonymized report forms in the OpenClinica web application. A unique code is generated for each individual and is not based on patient initials and birthdate. The principal investigator safeguards the key to the code. The handling of personal will comply with the Dutch Personal Data Protection Act (in Dutch: Wet Bescherming Persoonsgegevens [WBP]) and General Data Protection Regulation (GDPR) (in Dutch: Algemene verordening gegevensbescherming $[A V G]$ ). The project leader will keep the source data for 15 years. The investigators will have access to the final trial dataset; there are no contractual agreements that limit such access for the investigators. The datasets generated and/or analyzed during the current study are available from the corresponding author on reasonable request.

\section{Name and contact information for the trial sponsor}

Franciscus Gasthuis and Vlietland, represented by dr. T.M.A.L. Klem, sponsor Contact: phone: + 3110 4616289, e-mail: t.klem@franciscus.nl

Erasmus MC- Cancer Institute, represented by dr. L. Incrocci, co-sponsor Contact: phone: + 3110 7041507, email: l.incrocci@erasmusmc.nl

\section{Authors' contributions}

GS, JP, TK, EB, LK, GV, LK, JG, and IK conceived the study design. GS, JP, GV, $\mathrm{KV}$, and RB collected interventional data. Dosimetry analysis was performed by GS, JP, and JS. GS and JP wrote the manuscript. EB and GS provided the statistical analysis. All authors made contributions in revising the content, read, and approved the final manuscript. The results of this study will be published in high-impact peer-reviewed journals. It will be the responsibility of the Co-Pl of the study to write up the results within a year of its completion. Authorship eligibility guidelines will be adhering the International Committee of Medical Journal Editors (ICMJE) guidelines.

\section{Ethics approval and consent to participate}

The Erasmus MC Ethics Committee approved the original protocol of this study on 28 November 2016 (MEC-2016-400) and the last amendment was approved on 8 May 2018. All changes are included in this protocol. Any additional amendments will be notified to the METC as well as updated in the trial registry.

\section{Consent for publication}

Not applicable.

\section{Competing interests}

The authors declare that they have no competing interests.

\section{Publisher's Note}

Springer Nature remains neutral with regard to jurisdictional claims in published maps and institutional affiliations.

\section{Author details}

1Department of Surgery, Franciscus Gasthuis and Vlietland, PO Box 10900, 3004 BA Rotterdam, The Netherlands. ${ }^{2}$ Department of Radiation Oncology, Erasmus MC Cancer Institute, PO Box 5201, 3008 AE Rotterdam, The Netherlands. ${ }^{3}$ Department of Surgery, Erasmus MC Cancer Institute, PO Box 5201, 3008 AE Rotterdam, The Netherlands. ${ }^{4}$ Department of Statistics and Education, Franciscus Gasthuis and Vlietland, PO Box 10900, 3004 BA Rotterdam, the Netherlands. ${ }^{5}$ Department of Genetics, UMC Groningen, University of Groningen, P.O. Box 30001, 9700 RB Groningen, the Netherlands. 'Department of Radiology, Franciscus Gasthuis and Vlietland, PO Box 10900, 3004 BA Rotterdam, the Netherlands. ${ }^{7}$ Department of Radiation Oncology, Dalhousie University, 5820 University Avenue, Halifax, NS B3H1V7, Canada.

Received: 12 July 2018 Accepted: 1 November 2018

Published online: 17 December 2018

\section{References}

1. Peto R, Boreham J, Clarke M, Davies C, Beral V. UK and USA breast cancer deaths down 25\% in year 2000 at ages 20-69 years. Lancet. 2000;355(9217):1822.

2. SEER database. Cancer Stat Facts: Female Breast Cancer. https://seer.cancer. gov/statfacts/html/breast.html. Accessed on 12 July 2018.
3. Fisher B, Anderson S, Bryant J, Margolese RG, Deutsch M, Fisher ER, et al. Twenty-year follow-up of a randomized trial comparing total mastectomy, lumpectomy, and lumpectomy plus irradiation for the treatment of invasive breast cancer. N Engl J Med. 2002;347(16):1233-41.

4. Veronesi U, Cascinelli N, Mariani L, Greco M, Saccozzi R, Luini A, et al. Twenty-year follow-up of a randomized study comparing breast-consenving surgery with radical mastectomy for early breast cancer. N Engl J Med. 2002;347(16):1227-32.

5. Kim MK, Kim T, Moon HG, Jin US, Kim K, Kim J, et al. Effect of cosmetic outcome on quality of life after breast cancer surgery. Eur J Surg Oncol. 2015;41(3):426-32

6. Fisher ER, Sass R, Fisher B, Gregorio R, Brown R, Wickerham L. Pathologic findings from the National Surgical Adjuvant Breast Project (protocol 6). II. Relation of local breast recurrence to multicentricity. Cancer. 1986;57(9):1717-24.

7. Bethune WA. Partial breast irradiation for early breast cancer. J Natl Med Assoc. 1991;83(9):768-808

8. Strnad V, Ott OJ, Hildebrandt G, Kauer-Dorner D, Knauerhase H, Major T, et al. 5-year results of accelerated partial breast irradiation using sole interstitial multicatheter brachytherapy versus whole-breast irradiation with boost after breast-conserving surgery for low-risk invasive and in-situ carcinoma of the female breast: a randomised, phase 3, non-inferiority trial. Lancet. 2016; 387(10015):229-38.

9. Pignol JP, Caudrelier JM, Crook J, McCann C, Truong P, Verkooijen HA. Report on the clinical outcomes of permanent breast seed implant for early-stage breast cancers. Int J Radiat Oncol Biol Phys. 2015;93(3):614-21.

10. Vicini $F A$, Chen PY, Fraile M, Gustafson GS, Edmundson GK, Jaffray DA, et al. Low-dose-rate brachytherapy as the sole radiation modality in the management of patients with early-stage breast cancer treated with breastconserving therapy: preliminary results of a pilot trial. Int J Radiat Oncol Biol Phys. 1997;38(2):301-10.

11. White J, Winter K, Kuske RR, Bolton JS, Arthur DW, Scroggins T, et al. Longterm cancer outcomes from study NRG Oncology/RTOG 9517: A phase 2 study of accelerated partial breast irradiation with multicatheter brachytherapy after lumpectomy for early-stage breast cancer. Int J Radiat Oncol Biol Phys. 2016;95(5):1460-5.

12. NSABP. Protocol B-39/RTOG 0413, A Randomized Phase III Study of Conventional Whole Breast Irradiation (WBI) Versus Partial Breast Irradiation (PBI) for Women with Stage 0, I, or II Breast Cancer. http://www.nsabp.pitt. edu/B-39.asp. Accessed on 12 July 2018.

13. Vicini F, Winter K, Straube W, Wong J, Pass H, Rabinovitch R, et al. A phase $I / I$ trial to evaluate three-dimensional conformal radiation therapy confined to the region of the lumpectomy cavity for Stage I/I breast carcinoma: initial report of feasibility and reproducibility of Radiation Therapy Oncology Group (RTOG) Study 0319. Int J Radiat Oncol Biol Phys. 2005;63(5):1531-7.

14. Vaidya JS, Wenz F, Bulsara M, Tobias JS, Joseph DJ, Saunders C, et al. An international randomised controlled trial to compare TARGeted Intraoperative radioTherapy (TARGIT) with conventional postoperative radiotherapy after breast-conserving surgery for women with early-stage breast cancer (the TARGIT-A trial). Health Technol Assess. 2016;20(73):1-188,

15. Veronesi U, Orecchia R, Maisonneuve P, Viale G, Rotmensz N, Sangalli C, et al. Intraoperative radiotherapy versus external radiotherapy for early breast cancer (ELIOT): a randomised controlled equivalence trial. Lancet Oncol. 2013;14(13):1269-77.

16. Gitt A, Bose-Ribeiro H, Nieder C, Kup PG, Hermani H, Buhler $H$, et al. Treatment results of mammosite catheter in combination with whole-breast irradiation. Anticancer Res. 2016;36(1):355-60.

17. Yashar C, Attai D, Butler E, Einck J, Finkelstein S, Han B, et al. Strut-based accelerated partial breast irradiation: Report of treatment results for 250 consecutive patients at 5 years from a multicenter retrospective study. Brachytherapy. 2016;15(6):780-7.

18. Ott OJ, Strnad V, Hildebrandt G, Kauer-Dorner D, Knauerhase H, Major T, et al. GEC-ESTRO multicenter phase 3-trial: Accelerated partial breast irradiation with interstitial multicatheter brachytherapy versus external beam whole breast irradiation: Early toxicity and patient compliance. Radiother Oncol. 2016;120(1):119-23.

19. Polgar C, Ott OJ, Hildebrandt G, Kauer-Dorner D, Knauerhase H, Major T, et al. Late side-effects and cosmetic results of accelerated partial breast irradiation with interstitial brachytherapy versus whole-breast irradiation after breast-conserving surgery for low-risk invasive and in-situ carcinoma of the female breast: 5 -year results of a randomised, controlled, phase 3 trial. Lancet Oncol. 2017;18(2):259-68. 
20. Wobb JL, Shah C, Jawad MS, Wallace M, Dilworth JT, Grills IS, et al. Comparison of chronic toxicities between brachytherapy-based accelerated partial breast irradiation and whole breast irradiation using intensity modulated radiotherapy. Breast. 2015;24(6):739-44.

21. Mashouf S, Fleury E, Lai P, Merino T, Lechtman E, Kiss A, et al. Clinical significance of accounting for tissue heterogeneity in permanent breast seed implant brachytherapy planning. Int J Radiat Oncol Biol Phys. 2016;94(4):816-823.

22. Cuttino LW, Heffernan J, Vera R, Rosu M, Ramakrishnan VR, Arthur DW. Association between maximal skin dose and breast brachytherapy outcome: a proposal for more rigorous dosimetric constraints. Int J Radiat Oncol Biol Phys. 2011;81(3):e173-7.

23. Archambeau JO, Pezner R, Wasserman T. Pathophysiology of irradiated skin and breast. Int J Radiat Oncol Biol Phys. 1995;31(5):1171-85.

24. Vargo JA, Verma V, Kim H, Kalash R, Heron DE, Johnson R, et al. Extended (5-year) outcomes of accelerated partial breast irradiation using MammoSite balloon brachytherapy: patterns of failure, patient selection, and dosimetric correlates for late toxicity. Int J Radiat Oncol Biol Phys. 2014;88(2):285-91.

25. Chen PY, Vicini FA, Benitez P, Kestin LL, Wallace M, Mitchell C, et al. Longterm cosmetic results and toxicity after accelerated partial-breast irradiation. Cancer. 2006;106(5):991-9.

26. Ott OJ, Lotter M, Fietkau R, Strnad V. Accelerated Partial-Breast Irradiation with Interstitial Implants. Strahlenther Onkol. 2009;185(3):170-6.

27. Lilla C, Ambrosone CB, Kropp S, Helmbold I, Schmezer P, von Fournier D, et al. Predictive factors for late normal tissue complications following radiotherapy for breast cancer. Breast Cancer Res Treat. 2007;106(1):143-50.

28. Strnad V, Ott O, Potter R, Hildebrandt G, Hammer J, Resch A, et al. Interstitial brachytherapy alone after breast conserving surgery: interim results of a German-Austrian multicenter phase II trial. Brachytherapy. 2004;3(3):115-9.

29. Wazer DE, Kaufman S, Cuttino L, DiPetrillo T, Arthur DW. Accelerated partial breast irradiation: an analysis of variables associated with late toxicity and long-term cosmetic outcome after high-dose-rate interstitial brachytherapy. Int J Radiat Oncol Biol Phys. 2006;64(2):489-95.

30. Pignol JP, Rakovitch E, Keller BM, Sankreacha R, Chartier C. Tolerance and acceptance results of a palladium-103 permanent breast seed implant Phase I/II study. Int J Radiat Oncol Biol Phys. 2009;73(5):1482-8.

31. Correa C, Harris EE, Leonardi MC, Smith BD, Taghian AG, Thompson AM, et al. Accelerated Partial Breast Irradiation: Executive summary for the update of an ASTRO Evidence-Based Consensus Statement. Pract Radiat Oncol. 2017;7(2):73-9.

32. Polgar C, Van Limbergen E, Potter R, Kovacs G, Polo A, Lyczek J, et al. Patient selection for accelerated partial-breast irradiation (APBI) after breast -conserving surgery: recommendations of the Groupe Europeen de Curietherapie-European Society for Therapeutic Radiology and Oncology (GEC-ESTRO) breast cancer working group based on clinical evidence (2009). Radiother Oncol. 2010;94(3):264-73.

33. Pignol JP, Keller B, Rakovitch E, Sankreacha R, Easton H, Que W. First report of a permanent breast 103Pd seed implant as adjuvant radiation treatment for early-stage breast cancer. Int J Radiat Oncol Biol Phys. 2006;64(1):176-81.

34. Hoeller U, Tribius S, Kuhlmey A, Grader K, Fehlauer F, Alberti W. Increasing the rate of late toxicity by changing the score? A comparison of RTOG/EORTC and LENT/SOMA scores. Int J Radiat Oncol Biol Phys. 2003;55(4):1013-8.

35. Bentzen SM, Overgaard M. Relationship between early and late normal-tissue injury after postmastectomy radiotherapy. Radiother Oncol. 1991;20(3):159-65.

36. Common Terminology Criteria for Adverse Events v4.03 (CTCAE) Quick Reference: U.S.Department of Health and Human Services [updated June 14 2010. 4.03. https://www.eortc.be/services/doc/ctc/CTCAE_4.03_2010-06-14_ QuickReference_5x7.pdf. Accessed on 12 July 2018.

37. Cox JD, Stetz J, Pajak TF. Toxicity criteria of the Radiation Therapy Oncology Group (RTOG) and the European Organization for Research and Treatment of Cancer (EORTC). Int J Radiat Oncol Biol Phys. 1995;31(5):1341-6.

38. Stanton AL, Krishnan L, Collins CA. Form or function? Part 1. Subjective cosmetic and functional correlates of quality of life in women treated with breast -conserving surgical procedures and radiotherapy. Cancer. 2001;91(12):2273-81.

39. Struik GM, de Jongh FW, Birnie E, Pignol JP, Klem TM. Development and psychometric evaluation of a Dutch-translated shorter Breast Cancer Treatment Outcome Scale (Dutch BCTOS-13). Journal of Patient-Reported Outcomes, in press, https://doi.org/10.1186/s41687-018-0085-y.

40. Sprangers MA, Groenvold M, Arraras JI, Franklin J, te Velde A, Muller M, et al. The European Organization for Research and Treatment of Cancer breast cancer-specific quality-of-life questionnaire module: first results from a three-country field study. J Clin Oncol. 1996;14(10):2756-68.
41. Hilts M, Halperin H, Morton D, Batchelar D, Bachand F, Chowdhury R, et al. Skin dose in breast brachytherapy: Defining a robust metric. Brachytherapy. 2015;14(6):970-8.

42. Keller BM, Ravi A, Sankreacha R, Pignol JP. Permanent breast seed implant dosimetry quality assurance. Int J Radiat Oncol Biol Phys. 2012;83(1):84-92.

43. Pignol JP, Truong P, Rakovitch E, Sattler MG, Whelan TJ, Olivotto IA. Ten years results of the Canadian breast intensity modulated radiation therapy (IMRT) randomized controlled trial. Radiother Oncol. 2016;121(3):414-9.

44. Struik GM, Pignol JP, Kolkman-Deurloo IKK, Godart J, Verduijn GM, Koppert $L$, et al. Subcutaneous spacer injection to reduce skin toxicity in breast brachytherapy: a pilot study on mastectomy specimens. oral presentation. American Brachytherapy Society Annual Meeting; 8 June. San Francisco: Franciscus Gasthuis and Vlietland Erasmus MC- Cancer Institute; 2018.

\section{Ready to submit your research? Choose BMC and benefit from:}

- fast, convenient online submission

- thorough peer review by experienced researchers in your field

- rapid publication on acceptance

- support for research data, including large and complex data types

- gold Open Access which fosters wider collaboration and increased citations

- maximum visibility for your research: over $100 \mathrm{M}$ website views per year

At BMC, research is always in progress.

Learn more biomedcentral.com/submissions 